\title{
Study on the HIV drug resistance and its influencing factors in China Wang Chongjian*1,4, Li Yuqian² ${ }^{2}$ He Weihua ${ }^{3}$, Zhang Xiaodong ${ }^{3}$, Xu Yihua ${ }^{4}$ and Nie Shaofa ${ }^{4}$
}

\author{
Address: ${ }^{1}$ Department of Epidemiology and Biostatistics, School of Public Health, Zhengzhou University, Zhengzhou, 450001, PR China, \\ ${ }^{2}$ Department of Clinical Pharmacology, School of Pharmaceutical Science, Zhengzhou University, Zhengzhou, 450001, PR China, ${ }^{3}$ Center for \\ Disease Control and Prevention of Suizhou City, Suizhou, 441300, PR China and ${ }^{4}$ Department of Epidemiology and Biostatistics, School of Public \\ Health, Tongji Medical College, Huazhong University of Science \&Technology. Wuhan, 430030, PR China \\ * Corresponding author
}

from Frontiers of Retrovirology: Complex retroviruses, retroelements and their hosts

Montpellier, France. 21-23 September 2009

Published: 24 September 2009

Retrovirology 2009, 6(Suppl 2):P94 doi:I0.II86/1742-4690-6-S2-P94

This abstract is available from: http://www.retrovirology.com/content/6/S2/P94

(c) 2009 Chongjian et al; licensee BioMed Central Ltd.

\section{Objective}

To investigate HIV drug resistance and its influencing factors, and then provide scientific evidence for antiretroviral therapy and reducing drug resistance.

\section{Methods}

Questionnaires and blood sample collection for drug resistance detection were conducted among 109 treated cases and 84 non-treated cases. Viral load was detected by NASBA and drug resistance was measured by nested PCR.

\section{Results}

The viral load of 95 cases $(27$ treated cases and 68 nontreated cases) was more than 1000 copies $/ \mathrm{ml}$. The rate of drug resistance of treated and non-treated group was $40.74 \%$ and $10.29 \%$ respectively (see Table 1 ). The rate of different drug resistance was higher significantly in treated group than in no-treated group. However, the sensitive of different drug resistance was lower significantly in treated group than in no-treated group (see Table 2). Therapy schedule of 17 treated cases was stopped due to a variety of reasons, such as death, dizziness, liver damage, bellyache, et al (see Table 3). Except for five death cases, two suicides were discovered at 16 and 42 days respectively after stopping therapy.

\section{Conclusion}

Antiviral therapy can effectively reduce the viral load and inhibit viral replication. Drug resistance exists differently among treated and non-treated HIV/AIDS cases. HIV gene mutation may be the key reason for drug resistance.

Table I: The results of drug resistance detection in 95 cases with AIDS

\begin{tabular}{|c|c|c|c|c|}
\hline Group & No. & $\begin{array}{l}\text { Low-midrange resistance } \\
\text { (\%) }\end{array}$ & Height drug resistance (\%) & Total of resistance (\%) \\
\hline Stopping therapy & 12 & $0(0.00)$ & $3(25.00)$ & $3(25.00)$ \\
\hline Single therapy & 11 & $0(0.00)$ & $6(54.55)$ & $6(54.55)$ \\
\hline Therapeutic alliance & 4 & $0(0.00)$ & $2(50.00)$ & $2(50.00)$ \\
\hline No therapy & 68 & $6(8.82)$ & I (I.47) & $7(10.29)$ \\
\hline
\end{tabular}


Table 2: The results of resistance detection of different drugs among 95 cases

\begin{tabular}{|c|c|c|c|c|c|c|c|c|}
\hline \multirow[b]{2}{*}{ Group } & \multirow[b]{2}{*}{ Drug } & \multirow[b]{2}{*}{ No. } & \multirow[b]{2}{*}{ Sensitive (\%) } & \multicolumn{4}{|c|}{ Drug resistance } & \multirow[b]{2}{*}{ Total (\%) } \\
\hline & & & & $\begin{array}{l}\text { Low } \\
\text { resistance }\end{array}$ & $\begin{array}{l}\text { Midrange } \\
\text { resistance }\end{array}$ & $\begin{array}{l}\text { Height } \\
\text { resistance }\end{array}$ & $\begin{array}{l}\text { Latent } \\
\text { resistance }\end{array}$ & \\
\hline \multirow[t]{4}{*}{ Therapy } & DDI & 27 & $19(70.37)$ & 1 & I & 6 & 0 & $8(29.63)$ \\
\hline & D4T & 27 & $24(88.89)$ & 2 & I & 0 & 0 & $3(11.11)$ \\
\hline & EFV & 27 & $15(55.56)$ & 0 & 1 & 9 & 2 & $12(44.44)$ \\
\hline & NVP & 27 & $16(59.26)$ & 0 & 0 & 10 & 1 & II (40.74) \\
\hline \multirow{4}{*}{ No therapy } & DDI & 68 & $66(97.06)$ & 1 & 0 & 0 & 3 & $4(5.88)$ \\
\hline & D4T & 68 & $68(100.00)$ & 1 & 0 & 0 & 4 & $5(7.35)$ \\
\hline & $\mathrm{EFV}$ & 68 & $65(95.58)$ & 1 & 2 & 0 & 0 & $3(4.4 I)$ \\
\hline & NVP & 68 & $65(95.58)$ & 1 & 2 & I & 0 & $3(4.4 I)$ \\
\hline
\end{tabular}

Table 3: The reasons of stopping therapy

\begin{tabular}{|c|c|c|c|c|c|c|}
\hline $\begin{array}{l}\text { Course of } \\
\text { treatment } \\
\text { (Months) }\end{array}$ & Death & Liver damage & Dizziness & Skin damage & Bellyache & Total (\%) \\
\hline $0 \sim$ & 1 & I & 3 & - & - & $5(29.4 I)$ \\
\hline $\mathrm{I} \sim$ & 2 & 2 & I & 1 & I & 7 (4I.18) \\
\hline $2 \sim$ & 2 & - & I & - & - & $3(17.65)$ \\
\hline $\begin{array}{l}\ldots \\
7 \sim\end{array}$ & & I & & I & & $2(11.76)$ \\
\hline Total (\%) & $5(29.4 I)$ & $4(23.53)$ & $5(29.4 I)$ & $2(11.76)$ & I (5.88) & $17(100)$ \\
\hline
\end{tabular}

